\title{
Tests of discrete symmetries in positronium decays with the J-PET detector
}

\author{
Michał Silarski ${ }^{1, *}$ \\ for the J-PET Collaboration \\ ${ }^{1}$ Faculty of Physics, Astronomy and Applied Computer Science, Jagiellonian University, \\ S. Łojasiewicza 11, 30-348 Kraków, Poland
}

\begin{abstract}
As a relatively simple and purely leptonic state positronium constitutes a unique system to study discrete symmetries with precision limited only by the effects due to the weak interaction and photon-photon scattering. The experimental tests in the positronium decays were performed only on the $C, C \mathcal{P}$ and $C \mathcal{P T}$ symmetries with sensitivity much smaller than the predictions which opens a large window to search for phenomena beyond the Standard Model. In this article we present capability of the J-PET detector to improve the current precision of discrete symmetries tests in the decays of positronium atoms.
\end{abstract}

\section{Introduction}

Discrete symmetries of Parity $\mathcal{P}$, Charge Conjugation $C$ and Time Reversal $\mathcal{T}$ and their combinations, $C \mathcal{P}$ and $C \mathcal{P T}$ play an essential role in the modern particle physics. They prove to be very useful in the formulation of the Standard Model and calculation of many quantities, e.g. cross sections or decay rates, especially for the processes governed by the strong interaction. So far only the weak interactions were found to violate symmetries under the $\mathcal{P}$ [1], $C$ [2] and $\mathcal{T}[3,4]$ operators. A lot of effort was made to precisely measure the degree of $C P$ violation in the neutral kaons [5-7] and $B$ mesons system [8], but there are still several open issues under investigation. They are mainly related to rare $[9,10]$ and ultra rare kaon decays $[11,12]$, further $B$ meson studies and search for $C \mathcal{P}$ violation in $D$ meson decays. There is still no evidence of $C \mathcal{P}$ non-invariance in baryonic systems [8] and in the leptonic sector, in particular in the positronium decays [13-15] and in neutrino oscillations [16]. The $C P \mathcal{T}$ invariance is of a great importance since its violation would be an unambiguous sign of phenomena beyond the Standard Model. There have been performed many tests, including neutral kaons [10, 17-19], $B$ mesons [20] and positronium decays [21], and so far this symmetry seems to be exact for all the known interactions.

\section{Discrete symmetries tests with positronium atoms}

Positronium is a very promising system to test discrete symmetries and to look for physics beyond Standard Model. It is an eigenstate of the $\mathcal{P}$ operator (with eigenvalue $\lambda_{P}=-1$ ) and $C$ as well $\left(\lambda_{C}=(-1)^{S}\right)$. Thus, para-positronium $(p-P s)$, with spin $S=0$, is a $C \mathcal{P}$-odd

\footnotetext{
*e-mail: michal.silarski@uj.edu.pl
} 
state and ortho-positronium ( $o-P S$ ), with $S=1$, is even with respect to this operator. In case of positronium symmetries tests can be made with a very high precision limited, in principle, only by the effects due to the weak interaction, expected at $O\left(10^{-14}\right)$ [22], and photonphoton interaction at $O\left(10^{-9}\right)$ [23]. With positronium one can test the $C$ symmetry directly by searching for $o-P s$ decaying to an even number of gamma quanta and $p$ - $P s$ decays to an odd number of photons, respectively, which have opposite $C$ eigenvalues than the initial states. So far the following upper limits on branching ratios for both spin states were determined: $B R(o-P s \rightarrow 4 \gamma / o-P s \rightarrow 3 \gamma)<2.6 \cdot 10^{-6}$ at $90 \%$ C. L. [24], BR $(p-P s \rightarrow 3 \gamma / p-P s \rightarrow 2 \gamma)<$ $2.8 \cdot 10^{-6}$ at $68 \%$ C. L. [25], $B R(p-P s \rightarrow 5 \gamma / p-P s \rightarrow 2 \gamma)<2.7 \cdot 10^{-7}$ at $90 \%$ C. L. [26]. For $o-P s \rightarrow 3 \gamma$ decays one can also preform measurements of non-vanishing expectation values of operators which are odd under a certain symmetry transformation [27]. They can be constructed from momenta of the final-state photons $\overrightarrow{k_{1}}, \overrightarrow{k_{2}}$ and $\overrightarrow{k_{3}}$ in ascending order, their corresponding polarizations, $\epsilon_{1}, \epsilon_{2}, \epsilon_{3}$, and spin $(\vec{S})$ of the positronium [14]. So far the only experimental limits were set on the $C \mathcal{P}$ and $C \mathcal{P T}$ violation yielding the following mean values of corresponding operators: $\left\langle\vec{S} \cdot \overrightarrow{k_{1}}\left[\vec{S} \cdot\left(\overrightarrow{k_{1}} \times \overrightarrow{k_{2}}\right)\right]\right\rangle=0.0013 \pm 0.0012$ [15] and $\left\langle\vec{S} \cdot\left(\overrightarrow{k_{1}} \times \overrightarrow{k_{2}}\right)\right\rangle=0.0071 \pm 0.0062$ [21]. Determination of the spin requires usually a static magnetic field, which determined the quantization axis and mixes the para- and orthopositronium with vanishing spin projection. This shortens effectively the ortho-positronium lifetime and allows one to determine the spin polarization. The other way to determine the spin of $o-P s$ is using a $\beta^{+}$-emitter as the source of positrons for the positronium production. Due to the parity violation these positrons are polarized along their velocity direction. The polarization depends on the mean value of positrons velocity and is preserved to high extend during the positronium formation [28]. The full list of operators providing discrete symmetries tests can be found in Ref. [14]. Many of them, e.g. $\overrightarrow{k_{1}} \cdot \overrightarrow{\epsilon_{2}}$ or $\vec{S} \cdot \overrightarrow{\epsilon_{1}}$, require determination of the polarization of one of the final-state photons, which was not possible in the experiments performed so far.

\section{Discrete symmetries tests with the J-PET detector}

The J-PET experiment has a great potential to significantly improve the sensitivity of the discrete symmetries tests $[13,14,29,30]$. It was designed as a novel cost-effective and full-body PET scanner [31, 32], but this device can be used also for fundamental particle physics studies $[14,33]$. The J-PET detector is built out of 192 EJ-230 scintillator bars with dimensions $19 \times 7 \times 500 \mathrm{~mm}^{3}$ gathered in three coaxial layers, as it is presented in Fig. 1a). Each detection module is connected optically on both ends to a Hamamatsu R9800 photomultiplier. A novel frond-end electronics probes the photomultipliers signals at four threshold on both, leading and trailing edges, with accuracy of about 30 ps [34]. This enables also determination of the energy deposited by gamma quantum by measurement of the Time-Over-Threshold (TOT) [29]. An acquisition system used to collect and working in a continuous readout mode which allows true real-time tomographic data processing $[35,36]$. The principle of symmetries tests with the J-PET is shown schematically in Fig. 1b). As a positron source we use a ${ }^{22} \mathrm{Na}$ enclosed in a thin kapton foil and placed inside a vacuum chamber covered with a porous material (e.g. polymer, silica or aerogel). As it was mentioned positrons are, to high extend, longitudinally polarized and its spin direction is preserved with high probability during the $o-P s$ formation. Thus, determination of the ortho-positronium spin direction can be done knowing the source position and reconstructing the position of the positronium formation which at J-PET is done by a dedicated trilateration method [30]. The most probable effect of annihilation gamma quanta interaction with plastic scintillators is the Compton scattering which occurs most likely in the plane perpendicular to the electric vector of the photon. Thus, 

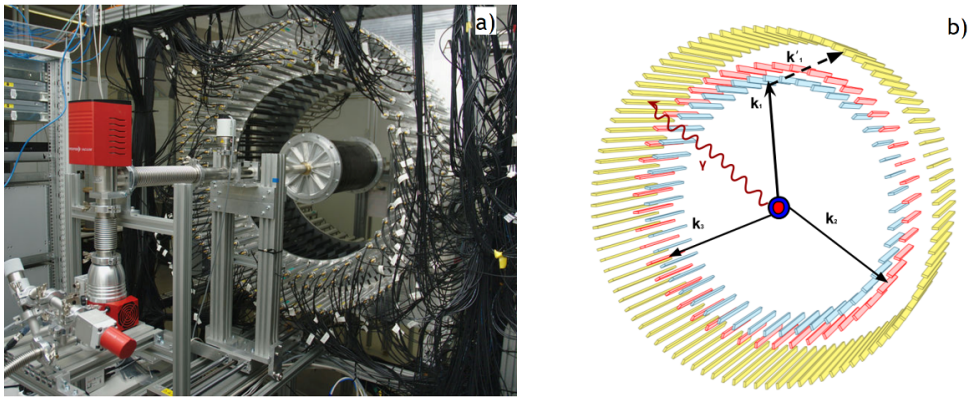

Figure 1. a) Photograph of the J-PET detector during data taking. A chamber used to create positronium in a porous material is installed together with a vacuum pump providing stable residual pressure. b) Schematic representation of the principle of ortho-positronium decays studies with the J-PET detector [14]. An open ${ }^{22} \mathrm{Na}$ source (red circle) is placed in the geometric center of the J-PET detector and surrouned by the porous target (in blue).The three gamma quanta from $o-P s \rightarrow 3 \gamma$ decay, with momenta $\overrightarrow{k_{1}}, \overrightarrow{k_{2}}, \overrightarrow{k_{3}}$, are registered by the J-PET detector. The measurement of $o$ - $P s$ lifetime is provided by the registration of the $\gamma$ quantum from the deexcitation of the ${ }^{22} \mathrm{Ne}$ isotope originating from the sodium $\beta^{+}$ decay. The registered momentum $\overrightarrow{k_{1}^{\prime}}$ of scattered photon can be used to determine its polarization.

measurement of four-momenta of annihilation and corresponding scattered quanta allows to determine the polarization of the primary quanta, e.g. by cross product: $\overrightarrow{\epsilon_{1}}=\overrightarrow{k_{1}} \times \overrightarrow{k^{\prime}}{ }_{1}[14]$ (see Fig. 1b). With the J-PET detector one can measure also the positronium lifetime which can be done using a gamma quantum from excited ${ }^{22} \mathrm{Ne}$ emitted soon after the positron. It can be distinguished from the annihilation and scattered photons via the TOT measurement which is much higher for deexcitation quanta. Lifetime measurement is used to separate the ortho- and para-positronium and in the Positron Annihilation Lifetime analysis which may be used in medical diagnostics $[37,38]$. The J-PET detector have been successfully commissioned and have been taking data with several different vacuum chambers and targets for $o$ - $P$ s formation and the first results of the $C$ and $C \mathcal{P}$ test are expected in 2019.

\section{Summary}

As the lightest purely charged leptonic state, positronium constitutes one of the best systems to search for new effects not included in the Standard Model. In this case the symmetries can be tested by searching for the forbidden decays, e.g. $p$-Ps $\rightarrow 3 \gamma$, or by measurement of expectation values of symmetry-odd operators. J-PET with the present detection setup and near future upgrades $[32,39,40]$ will be able to test discrete symmetries in the charged lepton system with significantly higher sensitivity than presently published results.

We acknowledge technical and administrative support of A. Heczko, M. Kajetanowicz and W. Migdał. This work was supported by The Polish National Center for Research and Development through grant INNOTECH-K1/IN1/64/159174/NCBR/12, the Foundation for Polish Science through the MPD and TEAM/2017-4/39 programmes, the National Science Centre of Poland through grants no. 2016/21/B/ST2/01222, 2017/25/N/NZ1/00861, the Ministry for Science and Higher Education through grants no. 6673/IA/SP/2016, 7150/E338/SPUB/2017/1 and 7150/E-338/M/2017, and the Austrian Science Fund FWF-P26783. 


\section{References}

[1] C. S. Wu et al., Phys. Rev. 105, 1413 (1957)

[2] R. L. Garwin et al., Phys. Rev. 105, 1415 (1957)

[3] A. Angelopoulos et al., Phys. Lett. B 444, 43 (1998)

[4] J. P. Lees et al., Phys. Rev. Lett. 109, 211801 (2012)

[5] G. Anzivino, J. Phys. Conf. Ser. 335, 012015 (2011)

[6] A. Alavi-Harati et al., Phys. Rev. D 67, 012005 (2003)

[7] A. Passeri, Nucl. Phys. Proc. Suppl. 181-182, 73 (2008)

[8] M. Tanabashi et al. (Particle Data Group), Phys. Rev. D 98, 030001 (2018)

[9] D. Babusci et al., Phys. Lett. B 723, 54-60 (2013)

[10] A. Anastasi et al., J. High Energy Phys. 09, 021 (2018)

[11] T. Yamanaka, Prog. Theor. Exp. Phys. 2012, $02 B 006$ (2012)

[12] M. Mirra, Nuovo Cimento C 038, 13 (2015)

[13] D. Kamińska et al., Eur. Phys. J. C 76, 445 (2016)

[14] P. Moskal et al., Acta Phys. Polon. B 47, 509 (2016)

[15] T. Yamazaki et al., Phys. Rev. Lett. 104, 083401 (2010)

[16] K. Abe et al., Phys. Rev. D 96, 092006 (2017)

[17] R. Adler et al., Phys. Lett. B 364, 239 (1995)

[18] D. Babusci et al., Phys. Lett. B 730, 89 (2014)

[19] F. Ambrosino et al., Phys. Lett. B 642, 315 (2006)

[20] R. Aaij et al., Phys. Rev. Lett. 116, 241601 (2016)

[21] P.A. Vetter, S.J. Freedman, Phys. Rev. Lett. 91, 263401 (2003)

[22] M. Skalsey, J. Van House, Phys. Rev. Lett. 67, 1993 (1991)

[23] M. Sozzi, Discrete Symmetries and CP Violation, (Oxford University Press, 2008)

[24] J. Yang et al., Phys. Rev. A 54, 1952 (1996)

[25] A.P. Mills, S. Berko, Phys. Rev. Lett. 18, 420 (1967)

[26] P.A. Vetter, S.J. Freedman, Phys. Rev. A 66, 052505 (2002)

[27] A. Gajos et al., arXiv:1804.07148 [physics.ins-det]

[28] P. Coleman, Positron Beams and Their Applications (World Scientific, 2000)

[29] P. Moskal et al., Nucl. Instrum. Meth. A 764, 317 (2014)

[30] A. Gajos et al., Nucl. Instrum. Meth. A 819, 54 (2016)

[31] S. Niedźwiecki et al., Acta Phys. Polon. B 48, 1567 (2017)

[32] P. Moskal et al., Phys. Med. Biol. 61, 2025 (2016)

[33] B. Hiesmayr, P. Moskal, Scientifc Reports 7, 15349 (2017)

[34] M. Pałka et al., JINST 12, P08001 (2017)

[35] G. Korcyl et al., Acta Phys. Polon. B 47, 491 (2016)

[36] G. Korcyl et al., IEEE Transactions on Medical Imaging, in press.

[37] B. Jasińska et al., Acta Phys. Polon. B 48, 1737 (2017)

[38] E. Kubicz et al., Nukleonika 60, 749 (2015)

[39] P. Moskal et al., arXiv:1805.11696 [physics.ins-det], submitted to Phys. Med. Biol.

[40] L. Raczyński et al., Phys. Med. Biol. 62, 5076 (2017) 